Design: The methodology comprised a quasi-experimental historical cohort design as blinding was not feasible with a nonintervention group (500) recruited first. The intervention group was recruited following a washout period. Interviewer administered questionnaires were completed at each time point and reported quitting validated by urinary cotinine measurement once in pregnancy (visit 2) and once after (visit 4).

Results: The overall proportion of responder and non-responder women who reported being current smokers at the first ante-natal visit was similar, however differences emerged within the groups with a higher proportion of the intervention group who were current smokers (56.8\%), being non-responders at visit 5, compared to the reference group $(41.8 \%)(p<0.05)$. Spontaneous quitters at the booking visit were also less likely to stay with the programme ( $61.4 \%$ vs. $38.6 \%$, $p<0.5)$, with no difference in effect for intervention and reference groups $(p=0.8)$. Fall off in response was an important element of the programme with $46.8 \%$ of the intervention group taking part at visit 5 compared to $53.2 \%$ in the reference group $(p=0.05)$.

Conclusion: These findings indicate that smoking status and intervention arm each affected participation, which illustrates the importance of estimating such a preference effect in assessing an effective health promotion programme.

\section{THE (AB)USE OF SYMPTOM SCORES IN ASTHMA CLINICAL TRIALS: EVIDENCE FROM A SYSTEMATIC REVIEW}

G Frampton, J Shepherd. Southampton Health Technology Assessments Centre (SHTAC), School of Medicine, University of Southampton, Epsilon House, Southampton Science Park, Southampton, UK

\section{doi:10.1136/jech.2009.096719q}

Background: The utility of patients' self-reported asthma symptom scores in clinical trials has not been critically assessed. We investigated the types of symptom scores used in asthma clinical trials and how they are analysed and interpreted.

Methods: Systematic reviews conducted to inform asthma management guidance in England and Wales were used as an evidence base. These reviews identified 87 randomised controlled trials of the effectiveness and safety of inhaled corticosteroids and long-acting $\beta$-agonists, published during 1985 to 2006. From these trials we extracted and appraised information on the characteristics of the symptom scales employed, and the way the symptom scores were interpreted and analysed.

Results: Most (78) of the asthma randomised controlled trials (90\%) reported the use of symptom scores, in all cases as secondary outcomes alongside measures of pulmonary function. Ten different numeric scales and 21 different classes of symptom combinations were identifiable among the 78 trials, which resulted in 44 unique symptom scoring scales. These scales could be further subdivided according to differences in the timing of symptom assessments and in the ways that the scores were coded. Only four (5\%) of the trials used validated scales. Lung function and asthma severity typically worsen at night but in all of 11 trials that reported both day and night symptom scores, the scores were consistently lower at night, irrespective of the numerical scale, study design, and asthma interventions involved. Asthma symptom scores appeared to be ordinal measures and in most trials were analysed parametrically, without reference to any assumptions about the type of distribution or equality of the scale intervals. In the asthma trials, interpretation always focused on changes in scores and the statistical significance, rather than on actual symptoms and the significance to patients.

Conclusions: Symptom scores are widely used in asthma RCTs in conjunction with estimates of pulmonary function but they lack validation and are interpreted inconsistently and uncritically. The numerical interpretation of scores seems detached from considering the real importance of symptoms to patients. Due to the large number of unique scales in use, it is questionable whether symptom scores can be meaningfully compared across studies, as is routinely attempted in meta-analyses. Adoption of a smaller set of validated symptom scales in asthma clinical trials could assist meta-analyses, improve understanding of how numeric scores reflect patient experiences, and enable a more thorough evaluation of the quantitative properties of the scales that are used.

\section{AN ANALYSIS OF THE STRUCTURE OF THE COMPONENTS OF METABOLIC SYNDROME USING MATROIDS}

${ }^{1}$ A Woolston, ${ }^{1} \mathrm{PD}$ Baxter, ${ }^{1} \mathrm{MS}$ Gilthorpe, ${ }^{2} \mathrm{E}$ Goodman, ${ }^{1} \mathrm{Y}-\mathrm{K}$ Tu. ${ }^{1}$ Centre for Epidemiology and Biostatistics, University of Leeds, Leeds,UK; ${ }^{2}$ Tufts Medical Center, Boston, MA, USA

\section{doi:10.1136/jech.2009.096719r}

Background: Metabolic syndrome (MetS) has been shown to be a risk factor for many chronic diseases, but the components of MetS are still controversial. In recent literature, exploratory and confirmatory factor analyses have been used to test the latent structure amongst MetS components and regression modelling is used to test the relation between chronic diseases and MetS components. The MetS components such as BMI, blood pressure and lipids are in general correlated and clustered, and this poses a challenge for statistical modelling. Collinearity amongst these components can have serious implications in regression analysis if not identified and treated with care. Whilst some exploratory analysis, such as principle component analysis (PCA), can provide an effective insight into the structure of the data, the results are often difficult to interpret to the non-statistician and lack the descriptive detail to explain effectively the clustering of the variables.

Methods: The approach we propose draws on a number of ideas in combinatorial mathematics and cluster analysis to generate a group of dependent subsets. The group is transformed into a matroid to ensure that the subsets adhere to the basic axioms of linear dependence. This allows the structure to be displayed in clear hierarchical form and provides an immediate interpretation of the clustering of the MetS components. We consider data from a paper by Shen (2003) in the American Journal of Epidemiology.

Results: The matroid technique identifies similar groups of dependent components to the factor analysis approach in the paper. These included: (1) glucose, PC glucose, insulin, PC insulin; (2) BMI, waist/hip ratio; (3) systolic BP, diastolic BP and (4) HDL and triglycerides. However, the matroid method additionally illustrates the dependencies at multiple collinearity thresholds. It shows the strengths of these dependencies and others, along with their location in the overall structure of the components of MetS. This reveals a concise depiction of the dependencies that the original factor analysis could not provide.

Conclusions: Presenting linear dependencies as subsets rather than latent variables gives the practitioner a greater choice of which variables to remove for regression analysis. This allows for an informed decision to be made about the inclusion of variables using clinical knowledge, as well as statistical reasoning, to limit the effects of collinearity in regression.

\section{Cancer II}

\section{HELP-SEEKING BEHAVIOUR IN SMOKERS AND EX-SMOKERS WITH SYMPTOMS OF LUNG CANCER. THE APPLICATION OF AN INTEGRATED MODEL}

S Smith, S Wyke, U Macleod, S Ziebland, N Campbell. Alliance for Self Care Research, University of Stirling, Stirling, UK

doi:10.1136/jech.2009.096719s

Introduction: Most patients with lung cancer in the UK are diagnosed when curative surgery is no longer an option and late 